\title{
Opinion Impact Models and Opinion Consensus Methods in Ad Hoc Tactical Social Networks
}

\author{
Demin Li, ${ }^{1}$ Jie Zhou, ${ }^{2}$ Jingjuan $\mathrm{Zhu}^{3}$ and Jiacun Wang ${ }^{4}$ \\ ${ }^{1}$ College of Information Science and Technology, Donghua University, Shanghai 201620, China \\ ${ }^{2}$ College of Science, Donghua University, Shanghai 201620, China \\ ${ }^{3}$ College of Public Administration, Nanjing Agricultural University, Nanjing 210095, China \\ ${ }^{4}$ Department of Computer Science and Software Engineering, Monmouth University, West Long Branch, NJ 07762, USA
}

Correspondence should be addressed to Demin Li; deminli@dhu.edu.cn

Received 1 August 2013; Accepted 15 August 2013

Academic Editor: Guanghui Wen

Copyright (c) 2013 Demin Li et al. This is an open access article distributed under the Creative Commons Attribution License, which permits unrestricted use, distribution, and reproduction in any medium, provided the original work is properly cited.

\begin{abstract}
Ad hoc social networks are special social networks, such as ad hoc tactical social networks, ad hoc firefighter social networks, and ad hoc vehicular social networks. The social networks possess both the properties of ad hoc network and social network. One of the challenge problems in ad hoc social networks is opinion impact and consensus, and the opinion impact plays a key role for information fusion and decision support in ad hoc social networks. In this paper, consider the impact of physical and logical distance on the opinions of individuals or nodes in heterogeneous social networks; we present a general opinion impact model, discuss the local and global opinion impact models in detail, and point out the relationship between the local opinion impact model and the global opinion impact model. For understanding the opinion impact models easily, we use the general opinion impact model to ad hoc tactical social networks and discuss the opinion impact and opinion consensus for ad hoc tactical social networks in the end.
\end{abstract}

\section{Introduction}

Ad hoc social networks are special social networks [1]. They possess both the properties of ad hoc networks and social networks, such as the properties of self-organization, decentralization, multihop communication, structure influence, opinion impact, and small world. The ad hoc social networks are applied in many fields, such as ad hoc tactical social networks [2], ad hoc firefighter social networks [3], and ad hoc vehicular social networks [4]. Lewenstein et al. [5] presented a social impact theory. Individuals are assumed to exchange, compare, adjust, and influence each other's attitudes. The total impact $I_{i}$ that the $i$ th individual experiences from his or her social environment is a function of the persuasive impact of those individuals who hold the opposite opinion to the $i$ th individual, relative to the supportive impact of those individuals who share the opinion.

In the paper $[5,6]$, the dynamics of the opinion is governed by the following rule:

$$
\sigma_{i}(t+1)=\operatorname{sign}\left[\sum_{j=1}^{N} d_{i j} \sigma_{j}(t) \xi_{j i}(t)+h_{i}(t)\right],
$$

where $d_{i j}$ denotes the connection between the nodes $i, j, \xi_{j i}(t)$ is the impact of the node $j$ on $i$, and $h_{i}(t)$ is the noise of the system. Considering the properties of ad hoc social networks, we improve the impact model as follows.

Huang et al. [6] assumed that in the social networks, each of the nodes holds one of the two opposite opinions denoted by $1,-1$. To illustrate the social impact of the community, the initial distributions of the opinion are as follows:

$$
\sigma_{i}(0)= \begin{cases}1, & i=1,2, \ldots, M, \\ -1, & i=M+1, \ldots, N,\end{cases}
$$

where $\sigma_{i}(t)$ denotes the opinion of the $i$ th node at the time step $t$ and $\sigma_{i}(0)$ denotes the opinion of the $i$ th node at the time step 0 . We know that in real ad hoc social networks, the opinion $\sigma_{i}(t)$ of $i$ may not be 1 or -1 and may be a fuzzy opinion or fuzzy number, such as triangular fuzzy number. For example, use $[-0.1,0,0.1]$ to denote the opinion of cross-bencher. So, in this paper, we always assume that the opinion of every node is a triangular fuzzy number, and let $\sigma_{i}(t)=\left[a_{i}(t), b_{i}(t), c_{i}(t)\right]$. Where $a_{i}(t), b_{i}(t)$, and $c_{i}(t)$ are all real numbers and satisfy $a_{i}(t) \leq b_{i}(t) \leq c_{i}(t)$. 
Therefore, $\sigma_{i}(t)=\left[a_{i}(t), b_{i}(t), c_{i}(t)\right]$ means that the individual or node $i$ 's opinion is between in $a_{i}(t)$ and $c_{i}(t)$ and centered in $b_{i}(t)$.

For ad hoc social networks, especially for scale-not-free social networks, the connection $d_{i j}$ may not be simply 1 or 0 but should be approximately inversely proportional to the physical distance or the hop number of communication between $i$ and $j$. In other words, the smaller the hop number is, the stronger the connection between $i$ and $j$ is. Considering the change of dynamic topology with the time in ad hoc social networks, the physical location or distance also change; in this paper, we assume that $d_{i j}$ is a function of time and $d_{i j}(t)=1 /\left(h_{i j}(t)+1\right)$, where $h_{i j}(t)$ is the hop number of communication from $i$ to $j$ at time $t$.

Ad hoc networks are usually assumed to be homogeneous, where each node shares the same radio capacity. However, homogeneous ad hoc networks suffer from poor scalability. Recent research has demonstrated its bottle neck performance through both theoretical analysis and simulation experiments and test bed measurements. Xu et al. [7] presented a design methodology to build a hierarchical largescale ad hoc network using different types of radio capabilities at different layers. In such a structure, nodes are dynamically grouped into multihop clusters. Each group elects a clusterhead to be a backbone node (BN). Then, higher-level links are established to connect the BNs into a backbone network. The backbone nodes have stronger social power or impact than the others. So, the backbone nodes have stronger opinion impact on the others. Illuminated in $[8,9]$, we use the social power factor $S P_{j}=\left(C_{j}\right)^{\alpha}$ to describe social power strength, where $C_{j}$ is the centrality of node $j$ and $\alpha$ is a parameter controlling the social diversity. So, for $\xi_{j i}(t)$ in (1), considering the properties of ad hoc social networks, it may be approximately proportion to social power factor $S P_{j}=\left(C_{j}\right)^{\alpha}$ and inversely proportional to the logical distance or level distance between node $j$ and $i$. So, we may define $\xi_{j i}(t)=S P_{j} /(|\operatorname{level}(j)-\operatorname{level}(i)|+1)$, where level $(i)$, level $(j)$ denote the levels of the node $i, j$ live in respectively.

By above discussion, (1) can be rewritten as follows:

$$
\sigma_{i}(t+1)=\frac{1}{N} \sum_{j=1}^{N} \frac{1}{h_{i j}(t)+1} \frac{S P_{j}}{|\operatorname{level}(j)-\operatorname{level}(i)|+1} \sigma_{j}(t) .
$$

We make some remarks to easily understand (3).

Remark 1. In (3), physical distance is a Euclid distance between node $i$ and $j$. For easy calculation, usually the physical distance is measured by the hop number of communication between $i$ and $j$. In other words, the hop number is approximately the number of nodes which connect node $i$ to node $j$, and the logical distance in (3) is a level distance between node $i$ and $j$. For easy calculation, especially in hierarchical network with different levels, usually the logical distance is measured by different levels between node $i$ and node $j$.

Remark 2. The difference between the physical distance and logical distance is that the physical distance is only considering the communication distance and not considering the relationship between the nodes, but the logical distance is considering the relationship and the social power between the nodes especially in hierarchical network.

Remark 3. In (3), physical distance mainly reflects the influence between nodes in the same level, and logical distance mainly reflects the influence between nodes in different levels. The impact of logical distance in (3) acts as an amplifier to physical distance. The impact mainly emphasizes the influence of backbone nodes and social powerful nodes.

To the best of our knowledge, there are no any papers using physical distance and logical distance that discuss opinion impact model.

The rest of the paper is organized as follows. In Section 2, we present the local and global opinion impact models and point out that the local opinion impact model is part of the global opinion impact model. By applying the models to ad hoc tactical social networks, in Section 3, we discuss the opinion impact. In Section 4, we give the opinion consensus for ad hoc tactical social networks. And we conclude in Section 5.

\section{Local and Global Opinion Impact Models}

By the general opinion impact models in Section 1, in the following, we discuss the local opinion impact models for nodes in the same level and global opinion impact models for nodes in different levels in detail.

2.1. The Local Opinion Impact Model. In this subsection, we only consider local opinion consensus in the same level, and $\xi_{j i}(t)=S P_{j}$. Assume $N_{i}$ nodes in the same level with the node $i$, so (3) can be rewritten as

$$
\sigma_{i}(t+1)=\frac{1}{N_{i}} \sum_{j=1}^{N_{i}} \frac{\sigma_{j}(t) S P_{j}}{h_{i j}(t)+1}, \quad i=1,2, \ldots, N_{i}
$$

If we only consider the neighbor nodes of $i$, we have that $h_{i j}(t)=1$, and

$$
\sigma_{i}(t+1)=\frac{1}{2 N_{i}} \sum_{j=1}^{N_{i}} \sigma_{j}(t) S P_{j}
$$

If we only consider the neighbor nodes of $i$, and neighbors' neighbor of $i$ we have

$$
\sigma_{i}(t+1)=\frac{1}{2 N_{i}} \sum_{j=1}^{N_{i_{1}}} \sigma_{j}(t) S P_{j}+\frac{1}{3 N_{i}} \sum_{j=N_{i_{1}}+1}^{N_{i_{1}}+N_{i_{2}}} \sigma_{j}(t) S P_{j} .
$$


In general, assume that the maximum hop number is $k_{i}$ for node $i$ in the same level, then

$$
\begin{array}{r}
\sigma_{i}(t+1)=\frac{1}{2 N_{i}} \sum_{j=1}^{N_{i_{1}}} \sigma_{j}(t) S P_{j}+\frac{1}{3 N_{i}} \sum_{j=N_{i_{1}}+1}^{N_{i_{1}}+N_{i_{2}}} \sigma_{j}(t) S P_{j}+\cdots \\
+\frac{1}{\left(k_{i}+1\right) N_{i}} \sum_{j=N_{i_{1}}+N_{i_{2}}+\cdots+N_{i_{i_{i}-1}}+1}^{N_{i_{1}}+N_{i_{2}}+\cdots+N_{i_{k_{i}-1}}+N_{i_{k_{i}}}} \sigma_{j}(t) S P_{j},
\end{array}
$$

where $N_{i_{l}}$ are the number of neighbors of $i$. The physical distance between those neighbors and $i$ is $l$ hops, $l=$ $1,2, \ldots, k$, and $N_{i_{1}}+N_{i_{2}}+\cdots+N_{i_{k}}=N_{i}$.

2.2. The Global Opinion Impact Model. For global opinion impact, consider the different levels; from (3), we get that

$$
\begin{aligned}
& \sigma_{i}(t+1)=\frac{1}{N} \sum_{j=1}^{N} d_{i j}(t) \sigma_{j}(t) \xi_{j i}(t) \\
& =\frac{1}{N} \sum_{j=1}^{N_{i_{1}}} d_{i j}(t) \sigma_{j}(t) S P_{j} \\
& +\frac{1}{2 N} \sum_{j=N_{i_{l 1}}+1}^{N_{i_{1}}+N_{i_{l 2}}} d_{i j}(t) \sigma_{j}(t) S P_{j}+\cdots \\
& +\frac{1}{m N} \sum_{j=N_{i_{1}}+N_{i_{l 2}}+\cdots+N_{i_{l m-1}}+1}^{N_{i_{1}}+N_{i_{l}}+\cdots+N_{i_{l m-1}}+N_{i_{l m}}} d_{i j}(t) \sigma_{j}(t) S P_{j} \text {, }
\end{aligned}
$$

where $N_{i_{u}}$ is the number of neighbors of the node $i$. The logical distance between those neighbors and $i$ is $l_{u}$ hops, $u=1,2, \ldots, m$, and $N_{i_{11}}+N_{i_{l 2}}+\cdots+N_{i_{l m}}=N$. Here, $N_{i_{1}}=N_{i}$ in local opinion impact model. So, we can see easily that the local opinion impact model is part of the global opinion impact model.

\section{The Opinion Impact Models for Tactical Social Networks}

In Section 2, we discussed generally the local and global opinion impact models. In this section, we apply the results in Section 2 to the special ad hoc social networks, ad hoc tactical social networks. Lihui $\mathrm{Gu}$ et al. [2] discussed the characteristics of tactical environment and showed the architecture of multilevel heterogeneous ad hoc wireless networks with Unmanned Aerial Vehicles (UAVs). The hierarchical infrastructure (Figure 1) reflected the three layers, including level 1: ground ad hoc wireless networks; Level 2: ground embedded mobile backbone networks; and level 3: aerial mobile backbone networks.

In Figure 1, in level 1, there are three groups or clusters. The largest group, denoted by $G_{1}$, includes eight soldiers; the other two groups, denoted by $G_{2}$ and $G_{3}$, each includes five

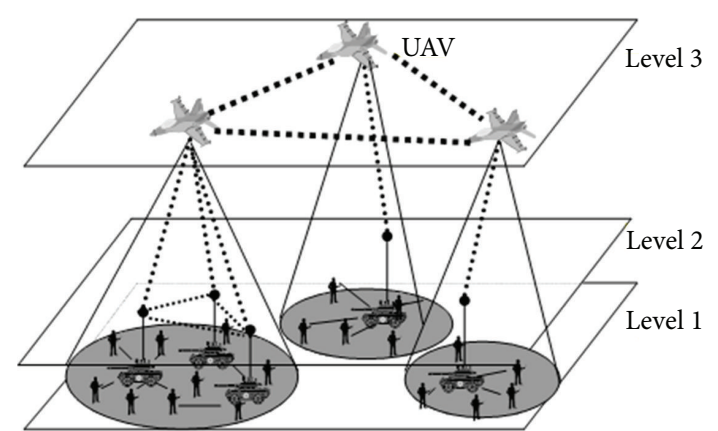

Figure 1: Multilevel UAV heterogeneous ad hoc wireless network [2].

soldiers. In level 2 , there are also three groups or clusters. The largest group includes three tanks, denoted by $G_{4}$; the other two groups, denoted by $G_{5}$ and $G_{6}$, each includes one tank. In level 3 , there is only one group, denoted $G_{7}$, which includes three planes. So, the total number of nodes is $N=18+5+$ $3=26$. For simplicity, we neglect the impact of centrality and discuss all situations as follows.

3.1. When $i$ Is in the Group $G_{1}$. From (8), we get

$$
\begin{aligned}
\sigma_{i}(t+1)= & \frac{1}{N} \sum_{j=1}^{N} \frac{\sigma_{j}(t)}{\left[1+h_{i j}(t)\right][\mid \text { level }(j)-\text { level }(i) \mid+1]} \\
= & \frac{1}{26} \sum_{j \in G_{1}} \frac{\sigma_{j}(t)}{\left[1+h_{i j}(t)\right]}+\frac{1}{26} \sum_{j \in G_{2}} \frac{\sigma_{j}(t)}{\left[1+h_{i j}(t)\right]} \\
& +\frac{1}{26} \sum_{j \in G_{3}} \frac{\sigma_{j}(t)}{\left[1+h_{i j}(t)\right]}+\frac{1}{52} \sum_{j \in G_{4}} \frac{\sigma_{j}(t)}{\left[1+h_{i j}(t)\right]} \\
& +\frac{1}{52} \sum_{j \in G_{5}} \frac{\sigma_{j}(t)}{\left[1+h_{i j}(t)\right]}+\frac{1}{52} \sum_{j \in G_{6}} \frac{\sigma_{j}(t)}{\left[1+h_{i j}(t)\right]} \\
& +\frac{1}{78} \sum_{j \in G_{7}} \frac{\sigma_{j}(t)}{\left[1+h_{i j}(t)\right]} .
\end{aligned}
$$

In (9), we discuss the situations as follows:

$$
\begin{aligned}
& j \in G_{1}, \quad \text { then } 2 \leq h_{i j}(t) \leq 3 \text { and } \\
& \quad \frac{1}{4} \sum_{j \in G_{1}} \sigma_{j}(t) \leq \sum_{j \in G_{1}} \frac{\sigma_{j}(t)}{\left[1+h_{i j}(t)\right]} \leq \frac{1}{3} \sum_{j \in G_{1}} \sigma_{j}(t) ; \\
& j \in G_{2}, \quad \text { then } h_{i j}(t)=5 \text { and } \\
& \sum_{j \in G_{2}} \frac{\sigma_{j}(t)}{\left[1+h_{i j}(t)\right]}=\frac{1}{6} \sum_{j \in G_{2}} \sigma_{j}(t) ; \\
& j \in G_{3}, \quad \text { then } h_{i j}(t)=5 \text { and } \\
& \sum_{j \in G_{3}} \frac{\sigma_{j}(t)}{\left[1+h_{i j}(t)\right]}=\frac{1}{6} \sum_{j \in G_{3}} \sigma_{j}(t) ;
\end{aligned}
$$


$j \in G_{4}$, then $1 \leq h_{i j}(t) \leq 2$ and

$$
\frac{1}{3} \sum_{j \in G_{4}} \sigma_{j}(t) \leq \sum_{j \in G_{4}} \frac{\sigma_{j}(t)}{\left[1+h_{i j}(t)\right]} \leq \frac{1}{2} \sum_{j \in G_{4}} \sigma_{j}(t) ;
$$

$j \in G_{5}$, then $h_{i j}(t)=4$ and

$$
\sum_{j \in G_{5}} \frac{\sigma_{j}(t)}{\left[1+h_{i j}(t)\right]}=\frac{1}{5} \sum_{j \in G_{5}} \sigma_{j}(t) ;
$$

$j \in G_{6}$, then $h_{i j}(t)=4$ and

$$
\sum_{j \in G_{6}} \frac{\sigma_{j}(t)}{\left[1+h_{i j}(t)\right]}=\frac{1}{5} \sum_{j \in G_{6}} \sigma_{j}(t) ;
$$

$j \in G_{7}, \quad$ then $2 \leq h_{i j}(t) \leq 3$ and

$$
\frac{1}{4} \sum_{j \in G_{7}} \sigma_{j}(t) \leq \sum_{j \in G_{7}} \frac{\sigma_{j}(t)}{\left[1+h_{i j}(t)\right]} \leq \frac{1}{3} \sum_{j \in G_{7}} \sigma_{j}(t) .
$$

Equation (9) follows as

$$
\begin{aligned}
\frac{1}{104} \sum_{j \in G_{1}} \sigma_{j}(t)+\frac{1}{156} \sum_{j \in G_{2} \cup G_{3}} \sigma_{j}(t)+\frac{1}{156} \sum_{j \in G_{4}} \sigma_{j}(t) \\
\quad+\frac{1}{260} \sum_{j \in G_{5} \cup G_{6}} \sigma_{j}(t)+\frac{1}{312} \sum_{j \in G_{7}} \sigma_{j}(t) \leq \sigma_{i}(t+1) \\
\leq \frac{1}{78} \sum_{j \in G_{1}} \sigma_{j}(t)+\frac{1}{156} \sum_{j \in G_{2} \cup G_{3}} \sigma_{j}(t)+\frac{1}{104} \sum_{j \in G_{4}} \sigma_{j}(t) \\
\quad+\frac{1}{260} \sum_{j \in G_{5} \cup G_{6}} \sigma_{j}(t)+\frac{1}{234} \sum_{j \in G_{7}} \sigma_{j}(t) .
\end{aligned}
$$
follows

In (11), we see that the impacting factors or weights are as

When node $j$ is in the group $G_{1}$, the impacting weight of $G_{1}$ is between $1 / 104$ and $1 / 78$; node $j$ is in $G_{2}$ and $G_{3}$, the impacting weights are $1 / 156$; node $j$ is in $G_{4}$, the impacting weight is between $1 / 156$ and $1 / 104$; node $j$ is in $G_{5}$ and $G_{6}$, the impacting weight is $1 / 260$; node $j$ is in $G_{7}$, the impacting weight is between $1 / 312$ and $1 / 234$.

3.2. When $i$ Is in Group $G_{2}$. Similarly, we can get the result as follows:

$$
\begin{aligned}
\frac{1}{78} \sum_{j \in G_{2}} \sigma_{j}(t) & +\frac{1}{156} \sum_{j \in G_{1} \cup G_{3}} \sigma_{j}(t)+\frac{1}{104} \sum_{j \in G_{5}} \sigma_{j}(t) \\
& +\frac{1}{260} \sum_{j \in G_{4} \cup G_{6}} \sigma_{j}(t)+\frac{1}{312} \sum_{j \in G_{7}} \sigma_{j}(t) \leq \sigma_{i}(t+1) \\
\leq & \frac{1}{78} \sum_{j \in G_{2}} \sigma_{j}(t)+\frac{1}{156} \sum_{j \in G_{1} \cup G_{3}} \sigma_{j}(t)+\frac{1}{104} \sum_{j \in G_{5}} \sigma_{j}(t) \\
& +\frac{1}{260} \sum_{j \in G_{4} \cup G_{6}} \sigma_{j}(t)+\frac{1}{234} \sum_{j \in G_{7}} \sigma_{j}(t) .
\end{aligned}
$$

3.3. When $i$ Is in Group $G_{3}$. Similarly, we can get the result as follows:

$$
\begin{aligned}
& \frac{1}{78} \sum_{j \in G_{3}} \sigma_{j}(t)+\frac{1}{156} \sum_{j \in G_{1} \cup G_{2}} \sigma_{j}(t)+\frac{1}{104} \sum_{j \in G_{6}} \sigma_{j}(t) \\
& +\frac{1}{260} \sum_{j \in G_{4} \cup G_{5}} \sigma_{j}(t)+\frac{1}{312} \sum_{j \in G_{7}} \sigma_{j}(t) \leq \sigma_{i}(t+1) \\
& \leq \frac{1}{78} \sum_{j \in G_{3}} \sigma_{j}(t)+\frac{1}{156} \sum_{j \in G_{1} \cup G_{2}} \sigma_{j}(t)+\frac{1}{104} \sum_{j \in G_{6}} \sigma_{j}(t) \\
& +\frac{1}{260} \sum_{j \in G_{4} \cup G_{5}} \sigma_{j}(t)+\frac{1}{234} \sum_{j \in G_{7}} \sigma_{j}(t) .
\end{aligned}
$$

\section{Opinion Consensus for the Ad Hoc Tactical Social Networks}

In this section, based on the opinion models in Section 3, we discuss the opinion consensus of soldiers in an ad hoc tactical social network for six situations in detail.

Situation 1. When $i \in G_{1}$ and $k \in G_{1}$, from (11), we transform the opinion consensus into vectors consensus, every components in a vector is the impacting weights of groups (from one to seven), respectively. So, the opinion consensus in time $t+1$ is

$$
\begin{aligned}
S\left[\sigma_{i}(t+1), \sigma_{k}(t+1)\right] & \\
=S & {\left[\left(\frac{1}{104}, \frac{1}{156}, \frac{1}{156}, \frac{1}{156}, \frac{1}{260}, \frac{1}{260}, \frac{1}{312}\right),\right.} \\
& \left.\left(\frac{1}{78}, \frac{1}{156}, \frac{1}{156}, \frac{1}{104}, \frac{1}{260}, \frac{1}{260}, \frac{1}{234}\right)\right] .
\end{aligned}
$$

By the vectors consensus definition of Cook and Seiford in [10], we have that the minimum opinion difference or opinion consensus between the node $i$ and $j$ is

$$
\begin{aligned}
& S\left[\sigma_{i}(t+1), \sigma_{k}(t+1)\right] \\
& =1-\left[\left(\frac{1}{78}-\frac{1}{104}\right)^{2}+\left(\frac{1}{104}-\frac{1}{156}\right)^{2}+\left(\frac{1}{234}-\frac{1}{312}\right)^{2}\right] .
\end{aligned}
$$

Let

$$
\begin{aligned}
\alpha= & \left(\frac{1}{234}-\frac{1}{312}\right)^{2}, \\
\beta= & \left(\frac{1}{156}-\frac{1}{104}\right)^{2}+\left(\frac{1}{78}-\frac{1}{156}\right)^{2} \\
& +\left(\frac{1}{260}-\frac{1}{156}\right)^{2}+\left(\frac{1}{104}-\frac{1}{260}\right)^{2}
\end{aligned}
$$

So, opinion consensus between the node $i$ and $k$ is

$$
1-\alpha \geq S\left[\sigma_{i}(t+1), \sigma_{k}(t+1)\right] \geq 1-\alpha-\beta .
$$


Situation 2. When $i \in G_{2}$ and $k \in G_{2}$, from (12), we transform the opinion consensus into vectors consensus, every components in a vector is the impacting weights of groups (from one to seven), respectively. So, the opinion consensus in time $t+1$, from we have

$$
S\left[\sigma_{i}(t+1), \sigma_{k}(t+1)\right]=1-\left[\left(\frac{1}{234}-\frac{1}{312}\right)^{2}\right]=1-\alpha
$$

Situation 3. When $i \in G_{3}$ and $k \in G_{3}$, from (13), we transform the opinion consensus into vectors consensus, every components in a vector is the impacting weights of groups (from one to seven), respectively. So, the opinion consensus in time $t+1$, from we have

$$
S\left[\sigma_{i}(t+1), \sigma_{k}(t+1)\right]=1-\left[\left(\frac{1}{234}-\frac{1}{312}\right)^{2}\right]=1-\alpha .
$$

Situation 4. When $i \in G_{1}$ and $k \in G_{2}$, or $i \in G_{2}$ and $k \in$ $G_{1}$, from (11) and (12), we transform the opinion consensus into vectors consensus, every components in a vector is the impacting weights of groups (from one to seven), respectively. So, the opinion consensus in time $t+1$ is

$$
\begin{array}{r}
S\left[\sigma_{i}(t+1), \sigma_{k}(t+1)\right] \\
=S\left[\left(\frac{1}{104}, \frac{1}{156}, \frac{1}{156}, \frac{1}{156}, \frac{1}{260}, \frac{1}{260}, \frac{1}{312}\right),\right. \\
\left.\quad\left(\frac{1}{156}, \frac{1}{78}, \frac{1}{156}, \frac{1}{260}, \frac{1}{260}, \frac{1}{104}, \frac{1}{234}\right)\right] .
\end{array}
$$

By the vectors consensus definition of Cook and Seiford, we have

$$
\begin{gathered}
S\left[\sigma_{i}(t+1), \sigma_{k}(t+1)\right] \\
=1-\alpha-\left[\left(\frac{1}{156}-\frac{1}{104}\right)^{2}+\left(\frac{1}{78}-\frac{1}{156}\right)^{2}\right. \\
\left.\quad+\left(\frac{1}{260}-\frac{1}{156}\right)^{2}+\left(\frac{1}{104}-\frac{1}{260}\right)^{2}\right], \\
S\left[\sigma_{i}(t+1), \sigma_{k}(t+1)\right]=1-\alpha-\beta .
\end{gathered}
$$

Situation 5. When $i \in G_{1}$ and $k \in G_{3}$, or $i \in G_{3}$ and $k \in$ $G_{1}$, from (11) and (13), we transform the opinion consensus into vectors consensus, every components in a vector is the impacting weights of groups (from one to seven), respectively. So, the opinion consensus in time $t+1$ is

$$
\begin{array}{r}
S\left[\sigma_{i}(t+1), \sigma_{k}(t+1)\right] \\
=S\left[\left(\frac{1}{104}, \frac{1}{156}, \frac{1}{156}, \frac{1}{156}, \frac{1}{260}, \frac{1}{260}, \frac{1}{312}\right),\right. \\
\left.\quad\left(\frac{1}{156}, \frac{1}{156}, \frac{1}{78}, \frac{1}{260}, \frac{1}{260}, \frac{1}{104}, \frac{1}{234}\right)\right] .
\end{array}
$$

By the vectors consensus definition of Cook and Seiford, we have

$$
S\left[\sigma_{i}(t+1), \sigma_{k}(t+1)\right]=1-\alpha-\beta .
$$

Situation 6. When $i \in G_{2}$ and $k \in G_{3}$, or $i \in G_{3}$ and $k \in$ $G_{2}$, from (12) and (13), we transform the opinion consensus into vectors consensus, every components in a vector is the impacting weights of groups (from one to seven), respectively. So, the opinion consensus in time $t+1$ is

$$
S\left[\sigma_{i}(t+1), \sigma_{k}(t+1)\right]=1-\left[\left(\frac{1}{234}-\frac{1}{312}\right)^{2}\right]=1-\alpha .
$$

We can see from the six situations that the opinion consensus of Situations 2, 3, and 6 is the strongest, the consensus strength is $1-\alpha$, the opinion consensus of Situations 4 and 5 is the weakest, the consensus strength is $1-\alpha-\beta$, the opinion consensus of Situation 1 is middle, and the consensus strength is between $1-\alpha$ and $1-\alpha-\beta$.

\section{Conclusions}

By using physical and logical distance, we present novel local and global opinion impact models. The models are suitable to general ad hoc social networks, and, for the first time, we improve the opinion impact model and establish foundation for group opinion consensus. For understanding the idea of the paper, we discuss opinion impact models and opinion consensus for ad hoc tactical social networks. Opinion impact model may influence the individual's information fusion and decision making in an ad hoc social networks that we are dealing with.

\section{Conflict of Interests}

We declare that there is no conflict of interests regarding the publication of this paper.

\section{Acknowledgments}

This work is partially supported by NSFC Grant no. 71171045; National ITER Plan Grant no. 2010GB108004; Shanghai Key Scientific Research Project under Grant no 05dz05036.

\section{References}

[1] P. Stuedi, O. Riva, and G. Alonso, "Demo abstract-ad hoc social networking using MAND," in Proceedings of 14th International Conference on Mobile Computing and Networking (MobiCom '08), September 2008.

[2] D. Lihui Gu, G. Pei, H. Ly, M. Gerla, and X. Hong, "Hierarchical routing for multi-layer ad-hoc wireless networks with UAVs," in Proceedings of the 21st Century Military Communications Conference Proceedings ( MILCOM '00), pp. 310-314, October 2000.

[3] Y. Chen, D. Li, C. Wang, and J. Wang, "Map synchronization and alternatives optimization for firefighters cooperative decision support in ad hoc networks," Journal of Networks, vol. 5, no. 1, pp. 39-46, 2010. 
[4] D. Li, Q. Li, J. Wang, and Z. Zhang, "Navigation function design for backbone connectivity in vehicle ad hoc networks," Computers \& Mathematics with Applications, vol. 61, no. 8, pp. 2067-2070, 2011.

[5] M. Lewenstein, A. Nowak, and B. Latané, "Statistical mechanics of social impact," Physical Review A, vol. 45, no. 2, pp. 763-776, 1992.

[6] G. Huang, J. Cao, G. Wang, and Y. Qu, "The strength of the minority," Physica A, vol. 387, no. 18, pp. 4665-4672, 2008.

[7] K. Xu, X. Hong, and M. Gerla, "Landmark routing in ad hoc networks with mobile backbones," Journal of Parallel and Distributed Computing, vol. 63, no. 2, pp. 110-122, 2003.

[8] G. Wen, Z. Duan, G. Chen, and X. Geng, "A weighted localworld evolving network model with aging nodes," Physica A, vol. 390, no. 21-22, pp. 4012-4026, 2011.

[9] M. Jalili, "Social power and opinion formation in complex networks," Physica A, vol. 392, no. 4, pp. 959-966, 2013.

[10] W. Cook and L. Seiford, "Priority ranking and consensus formation," Management Science, vol. 24, pp. 1721-1732, 1978. 


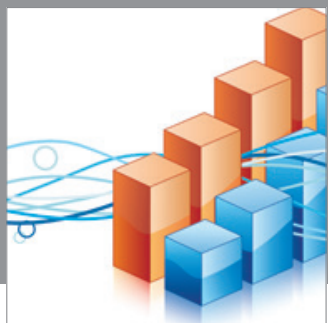

Advances in

Operations Research

mansans

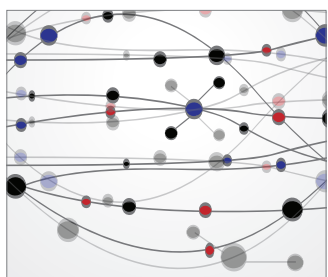

The Scientific World Journal
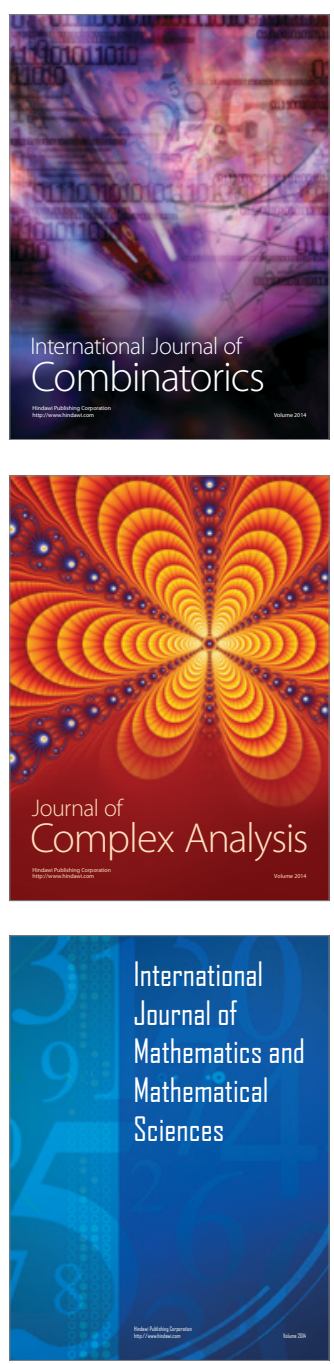
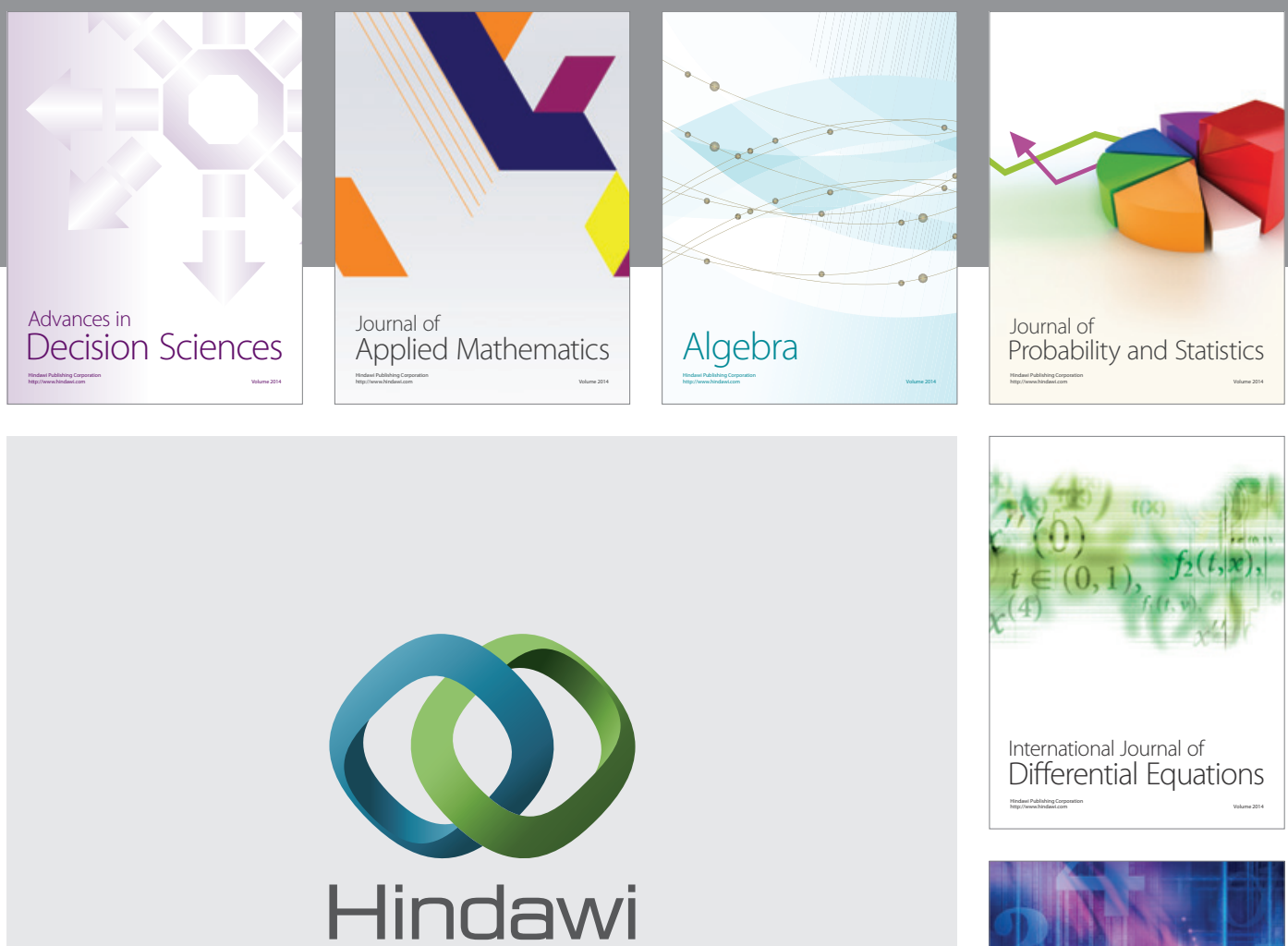

Submit your manuscripts at http://www.hindawi.com
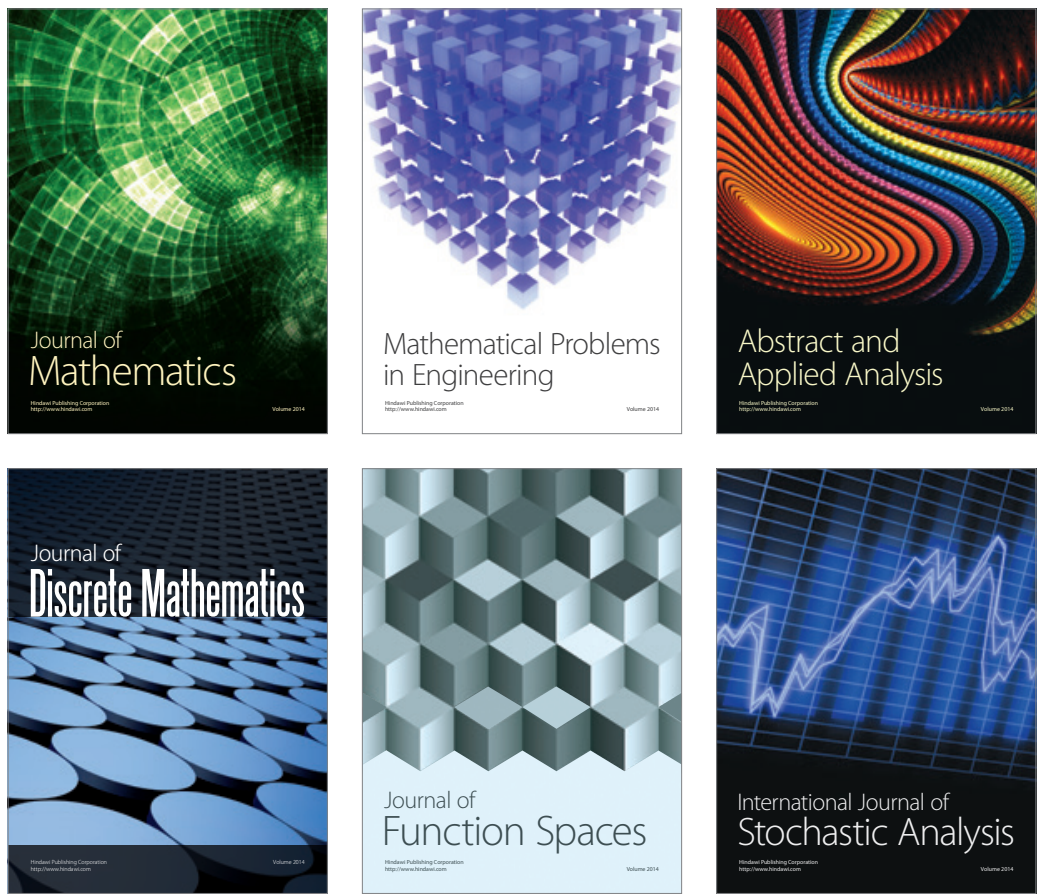

Journal of

Function Spaces

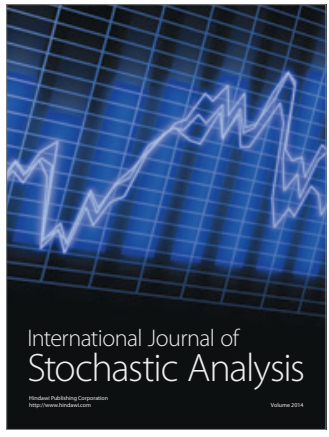

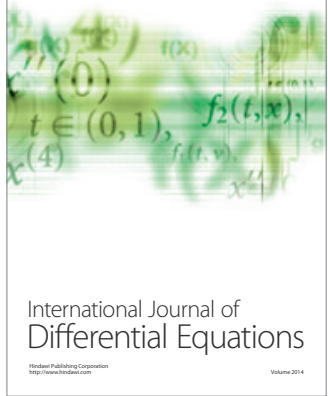
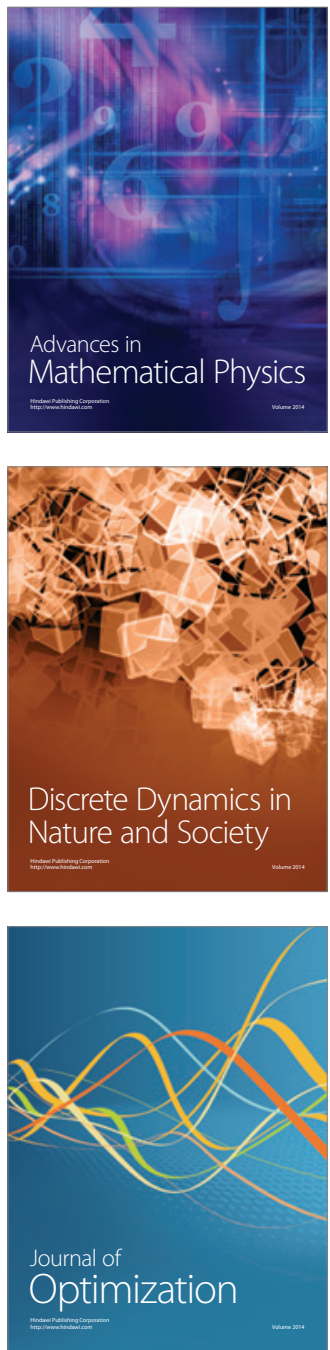